\title{
Tests of optical communications for deep space show promise
}

Kevin Birnbaum, William H. Farr, Jonathan Gin, Bruce Moision, Kevin J. Quirk, and Malcolm W. Wright

A free-space optical beam can reliably transfer more than one bit of data per photon received, even in the presence of background light.

Communication is vital for any spacecraft. After all, an instrument is of little use if it cannot return data. Space communications is currently dominated by radio-frequency (RF) links. Optical communication, however, promises higher data rates while adding less mass and volume to the spacecraft and consuming less power. Optical links have two main advantages over radio communications. First, the beam's shorter wavelength means less diffraction, so it spreads less as it travels through space and can be more tightly directed towards the receiver. Second, the optical system's available modulation bandwidth is much larger than that of an RF system, especially when considering the regulatory restrictions on RF allocation.

While spacecraft optical-communications systems have been demonstrated in Earth orbit, ${ }^{1-3}$ links to deep space at interplanetary distances have yet to be deployed. The improved optical-link performance is highly beneficial for these massand power-constrained spacecraft, yet the extreme distances involved pose a new set of challenges. For example, a link from Mars orbit to Earth would operate at up to 10,000 times longer range than one from geosynchronous orbit to the ground, corresponding to an additional loss of $80 \mathrm{~dB}$. Deep-space optical communications therefore require new paradigms and innovative technologies. 4

To reduce mission risk prior to flight operations, we have used elements of the Jet Propulsion Laboratory's deep-space optical-communications technology for a series of demonstrations aimed at validating subsystem models and operations. They evolved from initial in-fiber laboratory validations to current day-and-night operational free-space links that functionally validate transmitter and receiver systems in real time at data rates of over $44 \mathrm{Mbps}$ with efficiencies of approximately two bits per detected photon. ${ }^{5}$ We have also found that testing with pseudo-random data is not always sufficient to validate robust

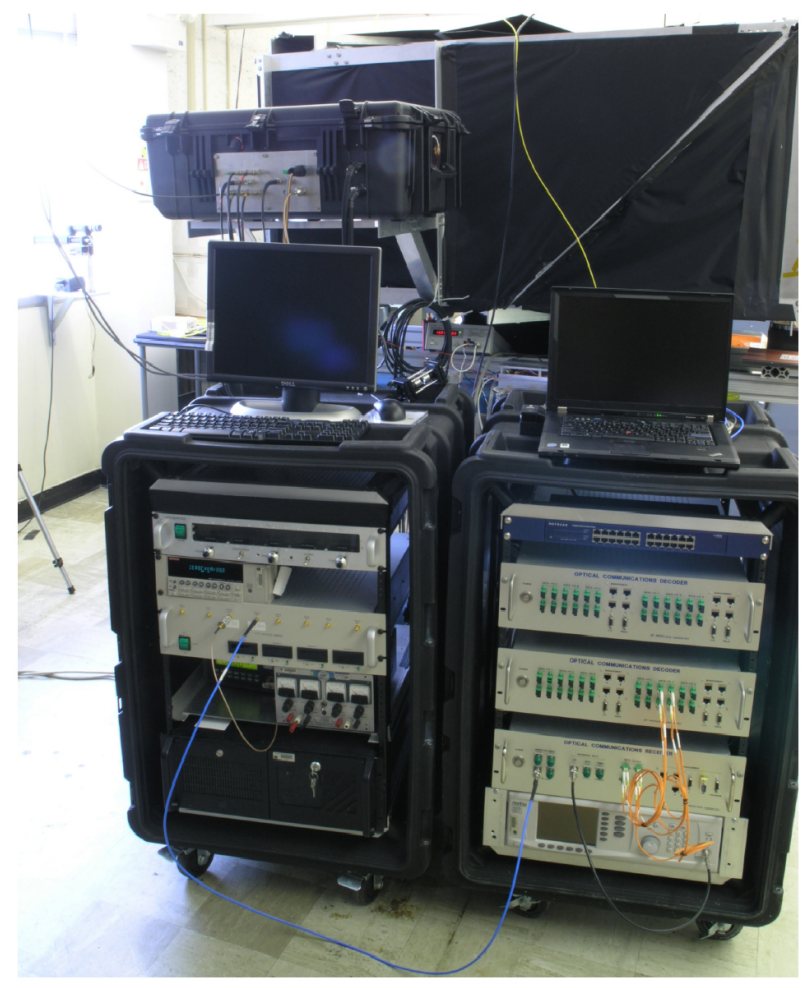

Figure 1. End-to-end testbed receiver. The receiver telescope (top) collects signal light through the sunlit window (top left). The digital receiver and decoder electronics are on the short rack to the right. Power supplies, amplifiers, and monitoring equipment are on the short rack to the left.

system operations, e.g., temporal acquisition and tracking issues may be masked by repetitive data sequences. Thus, we included a channel for live transmission of high-definition television at a compressed nominal data rate of $30 \mathrm{Mbps}$.

The testbed implements a building-top to building $100 \mathrm{~m}$ range link with the receiver placed indoors behind a window (see Figure 1). A custom telescope, used to test prototype low-cost, large-aperture optics, collects a small part of a wide

Continued on next page 
signal beam sent from the transmitter. The telescope's field of view allows us to vary the sky-background light level on the detector (depending on the sun angle). The link is typically operated under daytime conditions with suitable bandpass, spatial, and neutral-density filters to provide the desired detector signal and background levels that emulate a Mars-to-Earth link. The link achieves high photon efficiency using pulse-position modulation along with state-of-the-art error-correcting codes to encode multiple bits of information in each optical pulse's arrival time. Pulses are measured by a hybrid photodiode, a photon-counting detector, and the signal is processed by custom field-programmable gate-array-based hardware. The digital receiver and decoder hardware provide standard network interfaces and can be scaled up to gigabit-per-second data rates using a parallel approach.

Future work will include validating a prototype spacecraft terminal currently under development. A brassboard model, to be completed this year, has an innovative low-complexity design, ${ }^{6}$ a robust high-peak-power fiber laser, compact optics, and a novel vibration-isolation system. Once completed, the spacecraft terminal can be tested in a bidirectional link with the existing (or an upgraded) high-efficiency ground receiver. Planned improvements in the ground system include superconducting photoncounting detectors with higher efficiency and lower noise, and an active tracking and pointing system using feedback from the digital receiver. With successful tests minimizing the major risks in the end-to-end system, the promise of optical communications for deep space could soon be realized.

This research was carried out by the Jet Propulsion Laboratory, California Institute of Technology, under a contract with the National Aeronautics and Space Administration.

\section{Author Information}

Kevin Birnbaum, William H. Farr, Jonathan Gin, Bruce Moision, Kevin J. Quirk, and Malcolm W. Wright Jet Propulsion Laboratory (JPL)

Pasadena, CA

Kevin Birnbaum received a BS in physics from Stanford University in 1999 and a PhD in physics from the California Institute of Technology (Caltech) in 2005. His research interests include quantum optics, optical communications, and precision ranging.

William Farr attended Caltech from 1976 to 1980. He has traversed a variety of fields, including chemistry, neurobiology, electrical engineering, and computer science, resulting in publications in seven fields. He is now the technical lead of JPL's Optical Communications Technology Program of the Interplanetary Network Directorate.

Jonathan Gin received a BS in electrical engineering from the University of California at Los Angeles in 2002 and MS in electrical engineering from Stanford University in 2004. He is currently working on receiver development for optical-communications systems.

Bruce Moision received a BS in engineering from Harvey Mudd College in 1991 and a PhD in electrical engineering from the University of California at San Diego (UCSD) in 1999. He has been with JPL since 2001. His research interests include coding, modulation, and signal processing for optical communications.

Kevin Quirk received a BS in computer engineering in 1993, and MS and PhD degrees in electrical engineering from UCSD in 1997 and 2000, respectively. His research interests include communication theory, coding theory, and signal processing.

Malcolm Wright received his BSc from Victoria University in New Zealand and a PhD in physics from the University of New Mexico in 1992. Following semiconductor laser research at the Air Force Research Laboratory, he joined JPL in 1998 to develop laser transmitters for communication and ranging applications.

\section{References}

1. T. Tolker-Nielsen and G. Oppenhauser, In-orbit test result of an operational optical intersatellite link between ARTEMIS and SPOT4, SILEX, Proc. SPIE 4635, pp. 1-15, 2002. doi:10.1117/12.464105

2. T. Jono, Y. Takayama, N. Kura, K. Ohinata, Y. Koyama, K. Shiratama, Z. Sodnik, B. Demelenne, A. Bird, and K. Arai, OICETS on-orbit laser communication experiments, Proc. SPIE 6105, p. 610503, 2006. doi:10.1117/12.673751

3. B. Smutny, H. Kaempfner, G. Muehlnikel, U. Sterr, B. Wandernoth, F. Heine, U. Hildebrand, D. Dallmann, M. Reinhardt, A. Freier, R. Lange, K. Boehmer, T. Feldhaus, J. Mueller, A. Weichert, P. Greulich, S. Seel, R. Meyer, and R. Czichy, 5.6 Gbps optical intersatellite communication link, Proc. SPIE 7199, p. 719906, 2009. doi:10.1117/12.812209

4. H. Hemmati ed., Deep Space Optical Communications, JPL Deep Space Commun. Navig. Ser. 7, John Wiley and Sons, 2006. http://descanso.jpl.nasa.gov/ Accessed 28 May 2009.

5. K. Birnbaum, W. Farr, J. Gin, B. Moision, K. Quirk, and M. Wright, Demonstration of a high-efficiency free-space optical communications link, Proc. SPIE 7199, p. 71990A, 2009. doi: $10.1117 / 12.809720$

6. G. G. Ortiz, W. H. Farr, J. R. Charles, W. T. Roberts, V. Sannibale, J. Gin A. Saharaspude, and V. Garkanian, Canonical deep space optical communications transceiver, Proc. SPIE 7199, p. 71990K, 2009. doi:10.1117/12.812410 